\title{
Novel insights of HBV RNA in hepatitis B virus pathogenesis and clinical application
}

\author{
Wen-Bin Ding', Meng-Chao Wang', Jia-Ning Zhang', Da-Peng Sun'1, Jun-Peng Dong ${ }^{2}$, Wei-Ping Zhou', Fu \\ Yang $^{2}$
}

'The Third Department of Hepatic Surgery, Eastern Hepatobiliary Hospital, Naval Medical University, Shanghai 200438, China.

${ }^{2}$ Department of Medical Genetics, Naval Medical University, Shanghai 200433, China.

Correspondence to: Dr. Fu Yang, Department of Medical Genetics, Naval Medical University, 800 Xiangyin Road, Shanghai 200433, China. E-mail: yangfusq1997@smmu.edu.cn; Dr. Wei-Ping Zhou, The Third Department of Hepatic Surgery, Eastern Hepatobiliary Hospital, Naval Medical University, 225 Changhai Road, Shanghai 200438, China. E-mail: ehphwp3@126.com

How to cite this article: Ding WB, Wang MC, Zhang JN, Sun DP, Dong JP, Zhou WP, Yang F. Novel insights of HBV RNA in hepatitis B virus pathogenesis and clinical application. Hepatoma Res 2019;5:10. http://dx.doi.org/10.20517/2394-5079.2018.115

Received: 17 Dec 2018 First Decision: 10 Jan 2019 Revised: 20 Feb 2019 Accepted: 22 Feb 2019 Published: 2 Apr 2019

Science Editor: Guang-Wen Cao Copy Editor: Cai-Hong Wang Production Editor: Huan-Liang Wu

\begin{abstract}
Hepatitis B virus (HBV) infection is still a severe health problem in the world, and chronic hepatitis B (CHB) is the major cause of serious HBV-related complications, including fibrosis, hepatic failure, and hepatocellular carcinoma. It is difficult for $\mathrm{CHB}$ patients to achieve complete cure as the currently available antiviral drugs can hardly eradicate covalently closed circular DNA (cccDNA) in the infected liver. Since detecting intrahepatic cccDNA needs invasive procedure, it is urgent to find a noninvasive indicator to reflect the activity of cccDNA. Recently, growing numbers of studies have indicated that serum HBV RNA could be regarded as a new biomarker for CHB activity. In order to illustrate the molecular biology and clinical characteristics of HBV RNA, we systematically reviewed the latest research to summarize the role of HBV RNA in HBV replication and pathogenicity, and to better estimate its potential function as a remarkable biomarker in clinical application. Meanwhile, we will also point out the deficiencies of current research, and discuss the future direction of HBV RNA study.
\end{abstract}

Keywords: Hepatitis B virus, pregenomic RNA, serum biomarker, chronic hepatitis B, hepatocellular carcinoma, cirrhosis

\section{INTRODUCTION}

Hepatitis B virus (HBV) infection is one of the most common communicable diseases, with over 240 million people chronically infected all over the world ${ }^{[1]}$. Chronic hepatitis $\mathrm{B}(\mathrm{CHB})$ is the major etiological cause

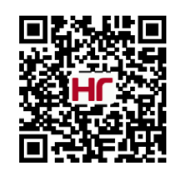


of developing serious liver complications, such as cirrhosis, hepatic failure, and primary hepatocellular carcinoma. Although the application of anti-HBV drugs [i.e., nucleos(t)ide analogues and pegylated interferon (peg-IFN)] can effectively suppress HBV replication and decrease the occurrence of corresponding complications, still more than 680,000 patients die of the fatal consequences of CHB every year ${ }^{[2]}$. The proportion of cirrhosis and liver cancer caused by HBV infection is about $30 \%$ and $45 \%$ worldwide. In China, the proportion is $60 \%$ and $80 \%$, which is much higher ${ }^{[3]}$. Therefore, it is urgent to acquire a better understanding of virus-host interaction to develop new therapeutics that increases the HBV cure rate.

HBV is an enveloped DNA virus which contains a $3.2 \mathrm{~kb}$ circular, partially double-stranded DNA genome. HBV establishes its genome as a covalently closed circular DNA (cccDNA) in the nucleus of the infected hepatocytes ${ }^{[4]}$. In the life cycle of HBV, the transcription of cccDNA to generate HBV mRNA is the beginning of $\mathrm{HBV}$ replication and the key factor for the continuous infection. Therefore, cccDNA is the most direct evidence of HBV infection and replication in vivo. Since the detection of cccDNA counts on biopsy, which is an invasive procedure, it is warrant to find some serum biomarkers that reflect the activity of intrahepatic cccDNA. The cccDNA is transcribed to 5 mRNAs during the viral replication. One of these transcripts, the pregenomic RNA (pgRNA) is not only the template for reversing transcription of viral DNA but also the coding mRNA for core protein and polymerase of $\mathrm{HBV}(\mathrm{pol})^{[5]}$. Recently, an increasing number of studies suggest that the HBV RNA can also be detected in serum. Circulating HBV RNA is HBV pgRNA and it may be used as a new serum biomarker for HBV infection, treatment and prognosis ${ }^{[6-8]}$. Thus, further comprehension of HBV pgRNA may provide new horizon for the better understanding the virushost interaction and the development of new HBV therapeutics. In this paper, we will review the current knowledge on the clinical significance of HBV RNA and its biological impact on host liver cells.

\section{THE ORIGIN AND NATURE OF HBV RNA}

The life cycle of HBV begins with the invasion of viral particles containing a 3.2-kb long partially double-stranded genome called relaxed circular DNA (rcDNA) into the hepatocytes through the sodium taurocholate co-transporting polypeptide (NTCP) receptor. The rcDNA is converted into cccDNA when coming into the nucleus ${ }^{[9]}$. The cccDNA plays a role as the transcription template for all the viral transcripts, involving the $3.5 \mathrm{~kb}$ pgRNA and precore mRNA (pre-C RNA), the $2.4 \mathrm{~kb}$ and $2.1 \mathrm{~kb}$ surface mRNAs, and a $0.7 \mathrm{~kb} \mathrm{X} \mathrm{mRNA}^{[10]}$. Among the $5 \mathrm{HBV}$ mRNA, pgRNA not only serves as the template for reverse transcription of HBV, but also is the template for translation of pol and core proteins ${ }^{[11]}$. The $5^{\prime}-\varepsilon$ region of pgRNA has the ability to combine with the pol. Once combined, they are packaged into viral capsid ${ }^{[12]}$. And inside the capsid, with the assist of the pol, pgRNA produces rcDNA through reverse transcription. Since newly created viral capsids can re-infect the nucleus, a small part of the newly formed rcDNAs re-enter into the nucleus to replenish the cccDNA pool. The remaining capsids are enveloped by the viral surface protein and released as Dane particles to infect new cells ${ }^{[13]}$. A recent research shows that HBV RNA can be detected in the serum of CHB patients, especially in those who have been taking antiviral drugs for a long time with their serum HBV DNA low or even undetectable. The research also revealed that serum HBV RNA is pgRNA which was presented in the virion-like particles, and serum pgRNA is produced by the transcription of cccDNA inside the hepatocytes ${ }^{[8]}$. The discovery of HBV RNA virion-like particle may complete the traditional life cycle of HBV infection. In theory, after the encapsidated pgRNAs get into hepatocytes, the reverse transcription to form rcDNA and cccDNA might be restarted, and this process eventually leads to HBV re-infection. However, more and stronger evidences are needed to prove the infection potential of HBV RNA virion-like particles.

\section{ROLES OF HBV RNA IN HBV-ASSOCIATED DISEASES}

HBV RNA not only is the template for both viral DNA reverse transcription and viral protein synthesis, but also plays a crucial role in the pathogenesis of HBV-associated diseases. Recently, many studies reveal 
that HBV RNA itself contributes to the progression of HBV-associated diseases through direct and indirect ways ${ }^{[14-23]}$. Nucleos(t)ide analogues (NAs) exerts its antiviral function through inhibiting the reverse transcription of HBV. As cccDNA is unaffected and its transcriptional activity remains, the formation of HBV RNAs continues. Therefore, in some CHB patients, although HBV DNA was maintained at extremely low level by anti-HBV drugs, serious HBV-related complications still occurred.

\section{HBV RNA and CHB}

Persistent infection of $\mathrm{HBV}$ is the main cause of $\mathrm{CHB}$, and continuous virus replication will eventually results in the inflammation and fibrosis of liver, which is the key characteristic of CHB. The effect of HBV RNA on HBV replication other than serving as reverse transcription template is poorly understood by now. However, HBV RNA may facilitate viral replication through deregulating the functions of host microRNAs. For example, one of the micro RNAs which is highly and specifically expressed in hepatocytes, miR-122, inhibits the replication of HBV in the liver. It has been reported that HBV RNA could act as sponges to bind and sequester endogenous miR-122, then the down-regulated expression or decreased function of miR-122 would increase level of cyclin G1, which further represses the expression of p53, leading to upregulation of HBV transcription via blocking specific combination of p53 with HBV enhancer elements ${ }^{[14,16]}$. It also has been reported that the miR-15 family might regulate HBV replication. The overexpression of the miR15 family members, miR-15a and miR-16-1, inhibits HBV replication. As HBV RNA can sequester these miRNAs, cyclin D1, the target of miR-15a and miR-16-1, is up-regulation, which makes a significant contribution HBV replication ${ }^{[17]}$. Furthermore, the viral-derived miRNA, miR-3, suppressed the transcription of pgRNA and HBc protein translation by targeting the $3.5-\mathrm{kb}$ transcript of $\mathrm{HBV}^{[18]}$. Yang et al ${ }^{[18]}$ thought that the inhibition of HBV replication might contribute to the development of persistent infection in $\mathrm{CHB}$ patients. However, more and further studies are need to verify his hypothesis.

\section{HBV RNA and hepatic fibrosis}

The cycle of continuous inflammation caused by viral replication and self-repairing of hepatocytes leads to the accumulation of extracellular matrix proteins, and eventually results in the development of fibrosis. The transforming growth factor- $\beta$ (TGF- $\beta$ ) pathway and nuclear factor- $\mathrm{k} B$ pathway together play an important role in the process of liver fibrosis. As mentioned above, miR-122 is down-regulated through sequestration caused by the role of HBV RNA as miRNA sponge in HBV-infected liver ${ }^{[14]}$. The altered expression of miR-122 activates the synthesis of collagen via the TGF- $\beta$ pathway, which participates in the liver fibrosis process ${ }^{[19]}$. Furthermore, the study of Sato et al.$^{[20]}$ indicated that the inflammatory factors induced by the $5^{\prime}-\varepsilon$ region of HBV pgRNA may aggravate the degree of inflammation and exacerbate the fibrosis of liver.

\section{HBV RNA and hepatocellular carcinoma}

Although the direct relationship between HBV RNA and the occurrence of hepatocellular carcinoma (HCC) has not been reported so far, the study of Wang et al ${ }^{[6]}$ showed that serum HBVRNA level correlates with the intrahepatic HBVRNA level, and serum HBVRNA level reflects the severity of histological changes and is associated with liver disease progression during NAs therapy, even in the CHB patients whose viral replication is suppressed. This means that serum HBV RNA is associated with the degree of intrahepatic inflammation and may be used as a new biomarker for reflecting hepatocarcinogenesis, especially in the CHB patients whose serum HBV DNA is suppressed by NAs therapy. It has also been reported that the 5 'region of HBV pgRNA induces the production of interferons and inflammatory cytokines in hepatocytes, which may lead to histological changes and the aggravation of fibrosis ${ }^{[20]}$. As we all know, the cirrhosis caused by liver fibrosis is a precancerous lesion of HCC.

These results together show that HBV RNA itself might promote the occurrence of HCC, at least to some extent. Moreover, the study of Halgand et al. ${ }^{[7]}$ showed that HBV pgRNA is detectable more frequently in HCC non-tumor tissues (90\%) than in HCC tumor tissues (67\%). When detectable in both compartments, the level of pgRNA was higher in non-tumor tissues than in tumor tissues. And the detection of pgRNA in 
tumour was correlated to the absence of tumorous microvascular invasion and better survival of patients. The results of microarrays and analysis of gene expression profiles showed that pgRNA positive HCCs were characterized by lower expression of cell cycle and DNA repair markers, and higher expression of the HBV receptor NTCP, which indicates a well-differentiated tumor. The replication of HBV in HCC may represent a sub-type of weakly invasive and hyper-differentiated $\mathrm{HCC}^{[7]}$. The possible mechanism of this phenomenon might be because the high metabolic status in poorly differentiated HCC is not suitable for the survival of HBV. Furthermore, it has also been reported that the circulating HBV RNA may be used as a biomarker for predicting the occurrence of $\mathrm{HCC}^{[21,22]}$.

HBV RNA, as miRNA sponge, can also promote the carcinogenesis of HCC by sequestration of host miRNAs. For example, HBV RNA could bind and sequester endogenous miR-122 through sponge adsorption, which upregulates PTTG1-binding protein and promotes the growth and invasion of $\mathrm{HCC}^{[14]}$. miRNA let-7 family is considered as tumor suppressors miRNAs. The expression of miRNA let-7 family is decreased in HCC, and it inhibits the progression of HCC via suppressing oncogenic targets, such as LIN28B, HMGA2 and c-Myc. Studies have demonstrated that let-7 family miRNAs (e.g., let-7a and let-7g) could be adsorbed and sequestered by HBV RNA, resulting in the promotion of tumorigenesis of HCC $^{[15,23]}$.

\section{CLINICAL SIGNIFICANCE OF HBV RNA}

In most CHB patients, the application of NAs potentially decreases HBV DNA and is associated with HBV induced complications. However, as the function of NAs is blocking HBV reverse transcription, the cccDNA is unaffected. The formation of pgRNA and the produce of HBV proteins would still continue in a long period of time. Hence cccDNA is the ultimate root of HBV replication. Since detecting cccDNA depends on liver biopsy, serum biomarkers reflecting the intrahepatic cccDNA activity are warranted. Giersch et al. ${ }^{[2]}$ found that levels of serum pgRNA significantly correlated with hepatocyte pgRNA levels in humanized uPA/SCID/beige (USB) mouse model of HBV infection treated with NAs and peg-IFN- $\alpha$, while in untreated HBV-infected mouses, serum pgRNA levels not only apparently correlated with hepatocyte pgRNA levels, but also clearly correlated with intrahepatic ccccDNA levels, indicating that serum pgRNA might serve as a useful clinical indicator to estimate the intrahepatic activity of cccDNA in HBV-infected patients ${ }^{[24]}$. Recently, more and more studies suggested that the pgRNA can be detected in serum and it may serve as a potent serum biomarker for reflecting the dynamic change of HBV replication.

\section{Evaluating the efficacy of CHB patients receiving NAs therapy}

During NAs therapy, it is important to monitor the dynamics of serum HBV DNA for assessing the virological response (VR) of CHB patients. According to clinical practice guidelines, VR is defined as serum HBV DNA being under the lowest limit of detection during NAs treatment, and that has been regarded as withdrawal indication of NAs therapy ${ }^{[3,25-27]}$. However, the virological rebound and hepatitis relapse often occurred when $\mathrm{CHB}$ patients discontinued the application of NAs. As mentioned above, the existence and transcription of cccDNA cannot be affected by NA. So merely detecting serum HBV DNA may not completely reflect the activity of cccDNA in CHB patients under NAs therapy. As HBV pgRNA can be detected in serum and its level reflects the transcriptional activity of intrahepatic cccDNA ${ }^{[6]}$, it is more valuable to detect both serum HBV pgRNA and serum HBV DNA than detecting serum HBV DNA alone when it comes to the better prediction of VR during NAs therapy. A growing number of studies have inferred that serum HBV RNA can be a useful marker for evaluating the efficacy of antiviral therapy ${ }^{[28-31]}$. Huang et al..$^{[31]}$ demonstrated that in CHB patients receiving NAs therapy, the low serum HBV RNA levels at week 12 of treatment could predict the initial VR. Another related study revealed that in HBeAg-positive CHB patients treated with NAs, baseline serum 3' full-length polyadenylated HBV RNA (flRNA) expression level could predict HBeAg seroconversion, and the decline of serum HBV RNA also showed a higher possibility of HBeAg seroconversion compared with HBV DNA and HBsAg during antiviral treatment ${ }^{[29]}$. 
Although these studies mentioned above showed that serum HBV RNA may have great potential to act as a supplementary biomarker for judging the effect of antiviral therapy, it remains unclear whether serum HBV RNA is superior to existing biomarkers, or whether it can replace other biomarkers for the same clinical applications.

\section{Monitoring safe discontinuation of NA-therapy in CHB patients}

As HBV cccDNA cannot be completely cleared during NAs therapy, most CHB patients have been suffering from virological rebound and HBV relapse, making it difficult to decide the timing of NAs therapy withdrawal. So, the majority of $\mathrm{CHB}$ patients have to receive NAs therapy for a long time, even their entire lifetime, which aggravates the financial burden for both the patients and the society ${ }^{[26,27]}$. As mentioned above, serum HBV RNA could be regarded as a potential indicator for cccDNA activity, so the vanishment of serum HBV RNA may represent the transcription silence of cccDNA. Therefore, serum HBV RNA could serve as a potential predictable marker for safe withdrawal of NAs therapy ${ }^{[24,32]}$. A study on $36 \mathrm{CHB}$ patients treated with NAs for at least 6 months revealed that after discontinuation of NA therapy for 24 weeks, their HBV DNA and HBV RNA titer on the third month of treatment was significantly associated with HBV DNA rebound and alanine aminotransferase rebound ${ }^{[33]}$. Another study of $33 \mathrm{CHB}$ patients who had received NAs treatment for at least 3 years and whose serum HBV DNA was undetectable afterwards showed that all patients with HBV RNA positive experienced virological rebound at the end of treatment after withdrawal of NAs for 24 weeks, while virological rebound occurred in only $25 \%$ of patients with negative serum HBV RNA ${ }^{[8]}$. However, as the sample size of these studies and the follow-up time are insufficient, additional studies with larger sample size and longer follow-up time are needed to further verify whether HBV RNA can be used as a predictive biomarker to reflect the rebound of HBV after discontinuation of antiviral treatment.

\section{To assess the prognosis of HBV-associated HCC}

Few studies have reported the relationship between HBV RNA and the prognosis of HBV-associated HCC. A recent study on $99 \mathrm{HBsAg}$-positive, virologically suppressed patients treated by tumour resection or liver transplantation indicated that HBV pgRNA was detectable more frequently in non-tumor (55/61; 90\%) than in tumor samples $(40 / 60(67 \%) ; P<0.01)$. When detectable in both compartments, the levels of pgRNA were slightly higher in non-tumor than in tumor samples. Moreover, the detection of pgRNA in HCC is significantly associated with lower incidence of vascular invasion and better survival rate. HCC expressing higher HBV pgRNA may represent a kind of well differentiated, less-proliferative and low-invasive HCC subtype $^{[7]}$. Therefore, HBV pgRNA might be used as a new biomarker for assessing the prognosis of HCC. However, in consideration of the insufficient sample size of this study, further research is still needed. Moreover, serum circulating HBV RNA may act as a biomarker for predicting the occurrence of HCC, which needs further study ${ }^{[21,22]}$.

\section{THE MEASUREMENT OF HBV RNA}

For the first time, Kock et al. ${ }^{[34]}$ successfully detected the HBV RNA in the serum of CHB patients through the method of rapid amplification of complementary DNA (cDNA)-ends (RACE) in 1996.The specific primer with a special anchored sequence was used to form cDNA after the extraction of HBV RNA from CHB patient serum. To ensure the high specificity for HBV RNA amplification, cDNA was amplified by PCR with HBV-specific forward primer and the reverse primer which is identical to the special anchored sequence. Since then, similar methods have been used to detect intrahepatic and serum HBV RNA in CHB patients ${ }^{[35,36]}$. Using unique primers designed for reverse transcription, Kairat et al.$^{[37]}$ developed RACEbased real-time quantitative PCR to specifically quantify serum 3' flRNA and 3' internally truncated polyadenylated HBV RNA later. Conventional RT-qPCR method with HBV-specific primers was also used to quantify intrahepatic and serum HBV RNA. However, DNase I pretreatment of the nucleic acids extracted is necessary to avoid DNA contamination before RT- $\mathrm{PPCR}^{[7,8]}$. Recently, super-sensitive droplet digital PCR was used to quantify serum HBV RNA by Wang et al. ${ }^{[6]}$ with HBV-specific primers. Collectively, many 
methods can be used to detect and quantify HBV RNA. As the widely accepted standardized method for HBV RNA detection is not available, further studies are needed to develop a more accurate and reliable technique of HBV RNA detection and quantification.

\section{FUTURE STRATEGY ON HBV RNA}

Before HBV RNA can be applied as a biomarker in clinic for CHB patients on a large scale, there are still many questions that need to be solved. Firstly, the methodology for detecting and quantifying serum HBV RNA should be standardized to make it possible that the results of different studies are comparable. Secondly, more clinical and molecular biology research is needed to further clarify details dynamics of HBV RNA under different conditions in CHB patients, for example, different HBV replication states, different stages of $\mathrm{CHB}$, and receiving what kind of antiviral drugs, NAs therapy or interferon therapy. Thirdly, more studies should focus on detecting HBV RNA among different ethnic groups and genotypes of CHB patients. Moreover, further and more exploration should be made to illuminate the correlation between HBV RNA and hepatocarcinogenesis.

\section{CONCLUSION}

In this review, we summarized the current progress and knowledge on the role of HBV RNA in HBV replication and pathogenicity. As mentioned above, HBV RNA may reflect the activity of intrahepatic cccDNA, even in CHB patients whose HBV DNA is maintained at low or undetectable levels through long-term antiviral therapy. And HBV RNA might play an important role in viral replication, promoting cirrhosis, and hepatocarcinogenesis. Moreover, serum HBV RNA has the potential of evaluating the efficacy of anti-viral drugs and predicting safe discontinuation of NA-therapy. And the intrahepatic HBV pgRNA could be used for assessing the prognosis of HCC. Therefore, HBV RNA possesses great potentials to be a new surrogate or complementary biomarker for HBV DNA in CHB patients. However, more research concerning the molecular biology of HBV RNA and more multi-centered and large-scale cohort studies should be conducted to assess and testify the feasibility and safety of HBV RNA as a novel biomarker for $\mathrm{CHB}$ in the future. Moreover, better understanding of HBV RNA will also provide new methods and strategies for anti-HBV therapy.

\section{DECLARATIONS}

\section{Authors' contributions}

Contributed the central idea and wrote the initial draft of this paper: Ding WB

Refining the ideas, carrying out the editing, revision, and finalizing of the manuscript: Zhou WP, Yang F

Discussed the ideas and did the literature research: Wang MC, Zhang JN, Sun DP, Dong JP

Writing and revisions of the manuscript: All authors

\section{Availability of data and materials}

Not applicable.

\section{Financial support and sponsorship}

This work was supported by National Key Basic Research Program of China (2014CB542102); National Key Research and Development Program of China (2016YFC1302303); National Human Genetic Resources Sharing Service Platform (2005DKA21300); Science Fund for Creative Research Groups, NSFC, China (81521091); State key infection disease project of China (2017ZX10203208); National Natural Science Foundation of China $(81502375,81472691,81672345)$.

\section{Conflicts of interest}

All authors declared that there are no conflicts of interest. 


\section{Ethical approval and consent to participate}

Not applicable.

\section{Consent for publication}

Not applicable.

\section{Copyright}

(c) The Author(s) 2019.

\section{REFERENCES}

1. Chisari FV, Isogawa M, Wieland SF. Pathogenesis of hepatitis B virus infection. Pathol Biol (Paris) 2010;58:258-66.

2. Chan SL, Wong VW, Qin S, Chan HL. Infection and cancer: the case of hepatitis B. J Clin Oncol 2016;34:83-90.

3. Chinese Society of Hepatology, Chinese Society of Infectious Diseases; Chinese Medical Association; Hou JL, Wei L. The guideline of prevention and treatment forchronic hepatitis B: a 2015 update. Chin J Hepato (Zhonghua GanZang Bing Za Zhi) 2015;23: 888905. (in Chinese)

4. Quasdorff M, Protzer U. Control of hepatitis B virus at the level of transcription. J Viral Hepat 2010;17:527-36.

5. Stahl M, Beck J, Nassal M. Chaperones activate hepadnavirus reverse transcriptase by transiently exposing a C-proximal region in the terminal protein domain that contributes to epsilon RNA binding. J Virol 2007;81:13354-64.

6. Wang J, Yu Y, Li G, Shen C, Meng Z, et al. Relationship between serum HBV-RNA levels and intrahepatic viral as well as histologic activity markers in entecavir-treated patients. J Hepatol 2018;68:16-24.

7. Halgand B, Desterke C, Rivière L, Fallot G, Sebagh M, et al. Hepatitis B virus pregenomic RNA in hepatocellular carcinoma: a nosological and prognostic determinant. Hepatology 2018;67:86-96.

8. Wang J, Shen T, Huang X, Kumar GR, Chen X, et al. Serum hepatitis B virus RNA is encapsidated pregenome RNA that may be associated with persistence of viral infection and rebound. J Hepatol 2016;65:700-10.

9. Hong X, Kim ES, Guo H. Epigenetic regulation of hepatitis B virus covalently closed circular DNA: implications for epigenetic therapy against chronic hepatitis B. Hepatology 2017;66:2066-77.

10. Block TM, Guo H, Guo JT. Molecular virology of hepatitis B virus for clinicians. Clin Liver Dis 2007;11:685-706.

11. Jones SA, Hu J. Hepatitis B virus reverse transcriptase: diverse functions as classical and emerging targets for antiviral intervention. Emerg Microbes Infect 2013;2:e56.

12. Stahl M, Beck J, Nassal M. Chaperones activate hepadnavirus reverse transcriptase by transiently exposing a C-proximal region in the terminal protein domain that contributes to epsilon RNA binding. J Virol 2007;81:13354-64.

13. Prange R. Host factors involved in hepatitis B virus maturation, assembly, and egress. Med Microbiol Immunol 2012;201:449-61.

14. Wang S, Qiu L, Yan X, Jin W, Wang Y, et al. Loss of microRNA 122 expression in patients with hepatitis B enhances hepatitis B virus replication through cyclin G(1) -modulated P53 activity. Hepatology 2012;55:730-41.

15. Deng M, Hou J, Hu J, Wang S, Chen M, et al. Hepatitis B virus mRNAs functionally sequester let-7a and enhance hepatocellular carcinoma. Cancer Lett 2016; 383:62-72.

16. Li C, Wang Y, Wang S, Wu B, Hao J, et al. Hepatitis B virus mRNA-mediated miR-122 inhibition upregulates PTTG1-binding protein, which promotes hepatocellular carcinoma tumor growth and cell invasion. J Virol 2013;87: 2193-205.

17. Wang Y, Jiang L, Ji X, Yang B, Zhang Y, et al. Hepatitis B viral RNA directly mediates down-regulation of the tumor suppressor microRNA miR-15a/miR-16-1 in hepatocytes. J Biol Chem 2013;288:18484-93.

18. Yang X, Li H, Sun H, Fan H, Hu Y, et al. Hepatitis B virus-encoded microRNA controls viral replication. J Virol 2017;91:01919-16.

19. Yin S, Fan Y, Zhang H, Zhao Z, Hao Y, et al. Differential TGF- $\beta$ pathway targeting by miR-122 in humans and mice affects liver cancer metastasis. Nat Commun 2016;7:11012.

20. Sato S, Li K, Kameyama T, Hayashi T, Ishida Y, et al. The RNA sensor RIG-I dually functions as an innate sensor and direct antiviral factor for hepatitis B virus. Immunity 2015,42:123-32.

21. Breitkreutz R, Zhang W, Lee M, Hoffmann A, Tokus M, et al. Hepatitis B virus nucleic acids circulating in the blood: distinct patterns in HBs carriers with hepatocellular carcinoma. Ann N Y Acad Sci 2001;945:195-206.

22. Hilger C, Velhagen I, Zentgraf H, Schroder CH. Diversity of hepatitis B virus X gene-related transcripts in hepatocellular carcinoma: a novel polyadenylation site on viral DNA. J Virol 1991;65:4284-91.

23. Takata A, Otsuka M, Ohno M, Kishikawa T, Yoshikawa T, et al. Mutual antagonism between hepatitis B viral mRNA and host microRNA let-7. Sci Rep 2016;6:23237.

24. Giersch K, Allweiss L, Volz T, Dandri M, Lutgehetmann M. Serum HBV pgRNA as a clinical marker for cccDNA activity. J Hepatol 2017;66:460-2.

25. Sarin SK, Kumar M, Lau GK, Abbas Z, Chan HL, et al. Asian-Pacific clinical practice guidelines on the management of hepatitis B: a 2015 update. Hepatol Int 2016;10:1-98.

26. Lampertico P, Agarwal K, Berg T, Buti M, Janssen HLA, et al; European Association for the Study of the Liver. EASL 2017 clinical practice guidelines on the management of hepatitis B virus infection. J Hepatol 2017;67:370-98.

27. Terrault NA, Bzowej NH, Chang KM, Hwang JP, Jonas MM, et al; American Association for the Study of Liver Diseases. AASLD guidelines for treatment of chronic hepatitis B. Hepatology 2016;63:261-83.

28. Jansen L, Kootstra NA, van Dort KA, Takkenberg RB, Reesink HW, et al. Hepatitis B virus pregenomic RNA is present in virions in 
plasma and is associated with a response to pegylated interferon Alfa-2a and uucleos(t)ide analogues. J Infect Dis 2016;213:224-32.

29. van Bommel F, Bartens A, Mysickova A, Hofmann J, Kruger DH, et al. Serum hepatitis B virus RNA levels as an early predictor of hepatitis B envelope antigen seroconversion during treatment with polymerase inhibitors. Hepatology 2015;61:66-76.

30. van Bommel F, van Bommel A, Krauel A, Wat C, Pavlovic V, et al. Serum HBV RNA as a predictor of peginterferon Alfa-2a (40KD) response in patients with HBeAg-positive chronic hepatitis B. J Infect Dis 2018;218:1066-74.

31. Huang YW, Takahashi S, Tsuge M, Chen CL, Wang TC, et al. On-treatment low serum HBV RNA level predicts initial virological response in chronic hepatitis B patients receiving nucleoside analogue therapy. Antivir Ther 2015;20:369-75.

32. Wang J, Du M, Huang H, Chen R, Niu J, et al. Reply to: "Serum HBV pgRNA as a clinical marker for cccDNA activity": Consistent loss of serum HBV RNA might predict the "para-functional cure" of chronic hepatitis B. J Hepatol 2017;66:462-3.

33. Tsuge M, Murakami E, Imamura M, Abe H, Miki D, et al. Serum HBV RNA and HBeAg are useful markers for the safe discontinuation of nucleotide analogue treatments in chronic hepatitis B patients. J Gastroenterol 2013;48:1188-204.

34. Kock J, Theilmann L, Galle P, Schlicht HJ. Hepatitis B virus nucleic acids associated with human peripheral blood mononuclear cells do not originate from replicating virus. Hepatology 1996;23:405-13.

35. Su Q, Wang SF, Chang TE, Breitkreutz R, Hennig H, et al. Circulating hepatitis B virus nucleic acids in chronic infection : representation of differently polyadenylated viral transcripts during progression to nonreplicative stages. Clin Cancer Res 2001;7:2005-15.

36. Hacker HJ, Zhang W, Tokus M, Bock T, Schroder CH. Patterns of circulating hepatitis B virus serum nucleic acids during lamivudine therapy. Ann N Y Acad Sci 2004;1022:271-81.

37. Kairat A, Beerheide W, Zhou G, Tang ZY, Edler L, et al. Truncated hepatitis B virus RNA in human hepatocellular carcinoma: its representation in patients with advancing age. Intervirology 1999;42:228-37. 